[20] E. Wong and B. Hajek, Stochastic Processes in Engineering Systems. New York: Springer-Verlag, 1985.

[21] T. Bielecki, D. Hernández-Hernández, and S. R. Pliska, "Value iteration for controlled markov chains with risk sensitive cost criterion," in Proc. 1999 Conf. Dec. Contr., 1999, pp. 126-130.

\section{Input-to-State Stability of Time-Delay Systems: A Link With Exponential Stability}

\author{
Nima Yeganefar, Pierdomenico Pepe, and Michel Dambrine
}

\begin{abstract}
The main contribution of this technical note is to establish a link between the exponential stability of an unforced system and the input-to-state stability (ISS) via the Liapunov-Krasovskii methodology. It is proved that a system which is (globally, locally) exponentially stable in the unforced case is (globally, locally) input-to-state stable when it is forced by a measurable and locally essentially bounded input, provided that the functional describing the dynamics in the unforced case is (globally, on bounded sets) Lipschitz and the functional describing the dynamics in the forced case satisfies a Lipschitz-like hypothesis with respect to the input. Moreover, a new feedback control law is provided for delay-free linearizable and stabilizable time-delay systems, whose dynamics is described by locally Lipschitz functionals, by which the closed-loop system is ISS with respect to disturbances adding to the control law, a typical problem due to actuator errors.
\end{abstract}

Index Terms-Exponential stability, input-to-state stability (ISS), Liapunov-Krasovskii theorem, nonlinear time-delay systems.

\section{INTRODUCTION}

For nondelayed systems, the input-to-state stability (ISS) property has been widely studied and its efficiency has been proved in practical applications such as networked control and robot manipulators (see, for a survey, [1]). The main point here is to focus on the robustness problem of nonlinear perturbed systems with possible large perturbations. ISS implies not only that the unperturbed system is asymptotically stable in the Liapunov sense but also that its behavior remains bounded when its inputs (e.g., exogenous perturbations) are bounded. This is due to the contribution of Sontag in [2], who was the first to harmonize the Liapunov state and the input-output approaches.

Recently, some authors have attempted to address the lack of results regarding time-delay systems. Until 2003, only the work [3] by Teel had been devoted to the ISS property. In Teel's paper, a definition of the ISS for time-delay systems was given and sufficient conditions were stated using a Razumikhin-type theorem. In [4], Pepe

Manuscript received June 8, 2007; revised November 14, 2007. Current version published August 29, 2008. The work of P. Pepe was supported by the Italian MIUR Project PRIN 2005. Recommended by Associate Editor S. Celikovsky.

N. Yeganefar is with the LAGIS UMR CNRS 8146, Ecole Centrale de Lille, BP 48, Cite Scientifique, 59651 Villeneuve-d'Ascq, France (e-mail: nimnima@gmail.com).

P. Pepe is with the Department of Electrical and Information Engineering, University of L'Aquila, 67040 Poggio di Roio, L'Aquila, Italy (e-mail: pepe@ing.univaq.it).

M. Dambrine is with the LAMIH UMR CNRS 8530, Université de Valenciennes, 59313 Valenciennes, France (e-mail: michel.dambrine@ univ-valenciennes.fr).

Digital Object Identifier 10.1109/TAC.2008.928340 and Jiang extended the definition of the ISS-Liapunov function to Liapunov-Krasovskii functional and presented a sufficient condition to guarantee the ISS property. Also, a recent paper by Liberzon [5] is devoted to the quantized approach and ISS using Teel's propositions.

The interest of the scientific community in the ISS property for time-delay systems is now rapidly increasing. In this context, we hope that this work will open even more perspectives with regard to this topic. Specifically, in this technical note, we will exhibit a link between exponential stability and the ISS property. Exponential stability has proved its efficiency in networked control. However, the influence of disturbances on the solutions behavior have to be more deeply analyzed from both a qualitative and a quantitative point of view. For networked control systems, the first work in this direction is [6] relying on Teel's results, which may be somewhat conservative due to the use of Razumikhin-Liapunov functions. Characterization of ISS for nonlinear time-delay systems is still a difficult task despite recent results [4], [7], [8].

We show in this technical note a link between ISS and exponential stability for a large class of systems. It is proved that a system which is (globally, locally) exponentially stable in the unforced case is (globally, locally) input-to-state stable when it is forced by a measurable and locally essentially bounded input, provided that the functional describing the dynamics in the unforced case is (globally, on bounded sets) Lipschitz and the functional describing the dynamics in the forced case satisfies a Lipschitz-like hypothesis with respect to the input. Moreover, a new feedback control law is provided for delay-free linearizable and stabilizable time-delay systems, whose dynamics is described by locally Lipschitz functionals, by which the closed-loop system is ISS with respect to disturbances adding to the control law, a typical problem due to actuator errors.

Notations: The symbol $|\cdot|$ stands for the Euclidean norm of a real vector or the induced Euclidean norm of a matrix. A measurable function $u:[0,+\infty) \rightarrow R^{m}, m$ positive integer, is said to be essentially bounded if $\operatorname{esssup}_{t>0}|u(t)|<+\infty$, where $\operatorname{ess~sup}_{t>0}|u(t)|=$ $\inf \{a \in[0,+\infty]: \lambda(\{\bar{t} \in[0,+\infty):|u(t)|>a\})=0\}, \lambda$ denoting the Lebesgue measure. The symbol $\|\cdot\|_{\infty}$ denotes the essential supremum norm, that is, for a measurable and essentially bounded

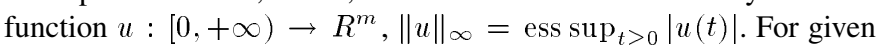
times $0 \leq T_{1}<T_{2}$, we indicate with $u_{\left[T_{1}, T_{2}\right)}:[0,+\infty) \rightarrow R^{m}$ the function given by $u_{\left[T_{1}, T_{2}\right)}(t)=u(t)$ for all $t \in\left[T_{1}, T_{2}\right)$ and $=0$ elsewhere. An input $u$ is said to be locally essentially bounded if, for any $T>0, u_{[0, T)}$ is essentially bounded. A function $w:[0, b) \rightarrow R$, $0<b \leq+\infty$, is said to be locally absolutely continuous if it is absolutely continuous in any interval $[0, c], 0<c<b$. A continuous function $\omega:[0, \infty) \rightarrow[0, \infty)$ is of class $\mathcal{K}$ if it is strictly increasing and $\omega(0)=0$ is of class $\mathcal{K}_{\infty}$ if it is of class $\mathcal{K}$ and is unbounded. A function $\beta:[0, \infty)^{2} \rightarrow[0, \infty)$ is of class $\mathcal{K} \mathcal{L}$ if for each fixed $t$ the function $s \rightarrow \beta(s, t)$ is of class $\mathcal{K}$ and for each fixed $s$ the function $t \rightarrow \beta(s, t)$ is nonincreasing and goes to zero as $t \rightarrow \infty$. For a given $\tau>0, \mathcal{C}$ denotes the vector space of continuous functions mapping the interval $[-\tau, 0]$ into $\mathrm{R}^{n}$ and for $\varphi \in \mathcal{C},\|\varphi\|_{c}=\sup _{-\tau<\theta \leq 0}|\varphi(\theta)|$. For a given positive real $H>0$, let $\mathcal{C}_{H}$ be the subset of $\mathcal{C}$ consisting of elements $\varphi$ whose norm $\|\varphi\|_{c}$ is bounded above by $H$. With the symbol $\|\cdot\|_{a}$ (see [4]), we indicate any seminorm in $\mathcal{C}$, such that, for some positive reals $\gamma_{a}$ and $\bar{\gamma}_{a}$, the following inequalities hold:

$$
\gamma_{a}|\phi(0)| \leq\|\phi\|_{a} \leq \bar{\gamma}_{a}\|\phi\|_{c} \quad \forall \phi \in \mathcal{C} .
$$

For any continuous function $x(s)$ defined on $-\tau \leq s<A, A>0$, and any fixed $t, 0 \leq t<A$, the standard symbol $x_{t}$ will denote the element of $\mathcal{C}$ defined by $x_{t}(\theta)=x(t+\theta),-\tau \leq \theta \leq 0$. 


\section{PRELIMINARIES}

\section{A. Exponential Stability of Unforced Systems}

We will consider the exponential stability problem for the following equation in $\mathrm{R}^{n}$ with bounded delay $\tau>0$ :

$$
\left\{\begin{array}{l}
\dot{x}(t)=f\left(x_{t}\right), \quad t \geq 0 \\
x_{0}=\psi
\end{array}\right.
$$

where $\psi \in \mathcal{C}$ and $f: \mathcal{C} \rightarrow \mathrm{R}^{n}$ is continuous and Lipschitz on bounded sets $f(0)=0$.

In this technical, we will denote by $x(t, \psi)$ (a vector of $\mathrm{R}^{n}$ ) the solution at time $t$ of system (2) with the initial condition $\psi$ at 0 . We will, by convenient abuse of notation, consider $x_{t}(\psi)$ (a function of $\mathcal{C})$ also a solution of (2). Observe that $x_{0}(\psi)=\psi$. We recall here the definition of exponential stability in the case of time-delay systems.

Definition 2.1: The solution $x(t)=0$ of (2) is exponentially stable if there exist positive reals $H, A$, and $B$ such that for every $\psi \in \mathcal{C}_{H}$ the solution $x_{t}(\psi)$ of (2) exists $\forall t \geq 0$ and furthermore satisfies

$$
\left\|x_{t}(\psi)\right\|_{c} \leq A e^{-B t}\|\psi\|_{c} .
$$

Definition 2.2: The solution $x(t)=0$ of (2) is globally exponentially stable if there exist positive reals $A$ and $B$ such that for every $\psi \in \mathcal{C}$ the solution $x_{t}(\psi)$ of (2) exists $\forall t \geq 0$ and furthermore satisfies

$$
\left\|x_{t}(\psi)\right\|_{c} \leq A e^{-B t}\|\psi\|_{c} .
$$

The next two theorems (here reported for the time-invariant case), proved in [9, Lemma 33.1], will play an important role in the proof of our main theorems in Section III.

Theorem 2.3: If system (2) is exponentially stable (with initial conditions in $\mathcal{C}_{H}, 0<H<+\infty$ ), then there exist a continuous functional $V(\varphi)$ defined on $\mathcal{C}_{(H / A)}$ and positive constants $C_{i}, i=1,2,3,4$, such that the following conditions hold $\forall \varphi, \xi \in \mathcal{C}_{(H / A)}$ :

$$
\begin{aligned}
C_{1}\|\varphi\|_{c} & \leq V(\varphi) \leq C_{2}\|\varphi\|_{c} \\
\limsup _{h \rightarrow 0^{+}} \frac{V\left(x_{h}(\varphi)\right)-V(\varphi)}{h} & \leq-C_{3}\|\varphi\|_{c} \\
|V(\varphi)-V(\xi)| & \leq C_{4}\|\varphi-\xi\|_{c} .
\end{aligned}
$$

Theorem 2.4: If system (2) is globally exponentially stable, then there exists a continuous functional $V(\varphi)$ defined on $\mathcal{C}$, which satisfies the following conditions in $\mathcal{C}$ :

$$
\begin{aligned}
C_{1}\|\varphi\|_{c} & \leq V(\varphi) \leq C_{2}\|\varphi\|_{c} \\
\limsup _{h \rightarrow 0+} \frac{V\left(x_{h}(\varphi)\right)-V(\varphi)}{h} & \leq-C_{3}\|\varphi\|_{c}
\end{aligned}
$$

where $C_{i}, i=1,2,3$, are some positive constants. Moreover, if the functional $f$ is globally Lipschitz, then there exists a positive real $C_{4}$, such that the following inequality holds $\forall \varphi, \xi \in \mathcal{C}$ :

$$
|V(\varphi)-V(\xi)| \leq C_{4}\|\varphi-\xi\|_{c} .
$$

Remark 1: In Theorems 2.3 and 2.4, the coefficients $C_{i}$, $i=1,2,3,4$, depend on the positive reals $A$ and $B$ and on the Lipschitz coefficient of the functional $f$ in $\mathcal{C}_{H}$ (or in $\mathcal{C}$ ) and can be easily computed (see [9]).

\section{B. Input-to-State Stability}

As previously stated, a definition of ISS for time-delay systems has been given in [3] and a useful characterization has been presented in [4]. For the reader's convenience, and to make our work self-contained, we report here the definition of ISS for time-delay systems and its characterization with an ISS Liapunov-Krasovskii functional (see [2] and [4]).
Consider the system

$$
\left\{\begin{array}{l}
\dot{x}(t)=f\left(x_{t}, u(t)\right), \quad t \geq 0 \\
x_{0}=\psi
\end{array}\right.
$$

where $f$ is a continuous functional defined on $\mathcal{C} \times \mathrm{R}^{m}$, Lipschitz on bounded sets $f(0,0)=0$. The input $u$ is a measurable and locally essentially bounded function of $t$ for all $t \geq 0$. Consider also the unforced system

$$
\left\{\begin{array}{l}
\dot{x}(t)=f\left(x_{t}, 0\right), \quad t \geq 0 \\
x_{0}=\psi
\end{array}\right.
$$

Definition 2.5: System (11) is said to be locally input-to-state stable if there exist two positive reals $r$ and $r_{u}$, a class $\mathcal{K} \mathcal{L}$ function $\beta$, and a class $\mathcal{K}$ function $\gamma$, such that, $\forall \psi \in C_{r}$ and $\forall u$ such that $\operatorname{ess} \sup _{t>0}|u(t)|<r_{u}$, the solution exists for all $t \geq 0$ and furthermore satisfies

$$
|x(t, \psi)| \leq \beta\left(\|\psi\|_{c}, t\right)+\gamma\left(\left\|u_{[0, t)}\right\|_{\infty}\right) .
$$

Definition 2.6: System (11) is said to be input-to-state stable if there exist a class $\mathcal{K} \mathcal{L}$ function $\beta$ and a class $\mathcal{K}$ function $\gamma$, such that, for any initial state $\psi$ and any locally essentially bounded input $u$, the solution $x_{t}(\psi)$ exists for all $t \geq 0$ and furthermore satisfies

$$
|x(t, \psi)| \leq \beta\left(\|\psi\|_{c}, t\right)+\gamma\left(\left\|u_{[0, t)}\right\|_{\infty}\right) .
$$

In the following, the continuity of a functional $V: \mathcal{C} \rightarrow R^{+}$is intended with respect to the supremum norm.

Given a continuous functional $V: \mathcal{C} \rightarrow R^{+}$, the upper-right hand Dini derivative (as proposed in [10] and used in [4] and [11] and, in a generalized version [12]) is given by

$$
D^{+} V(\varphi, v)=\limsup _{h \rightarrow 0^{+}} \frac{1}{h}\left(V\left(\varphi_{h, v}\right)-V(\varphi)\right)
$$

where $\varphi_{h, v} \in \mathcal{C}$ is given by

$$
\varphi_{h, v}(s)= \begin{cases}\varphi(s+h), & s \in[-\tau,-h) \\ \varphi(0)+(s+h) f(\varphi, v), & s \in[-h, 0] .\end{cases}
$$

It is proved in [13] that, under Caratheódory conditions, if the functional $V$ is locally Lipschitz, then, for any $\varphi \in \mathcal{C}$, almost everywhere in $t$

$$
D^{+} V\left(x_{t}(\varphi), u(t)\right)=\limsup _{h \rightarrow 0^{+}} \frac{V\left(x_{t+h}(\varphi)\right)-V\left(x_{t}(\varphi)\right)}{h} .
$$

Moreover, it is proved in [14] that the problem of the absolute continuity of the function $t \rightarrow V\left(x_{t}(\varphi)\right)$ (see the hypothesis $H p_{1}$ in [4]) is overcome if $V$ is locally Lipschitz.

Taking into account the above two facts, a main contribution in [4] is here reported by the following definitions and theorems.

Definition 2.7: A locally Lipschitz continuous functional $V: \mathcal{C} \rightarrow$ $\mathrm{R}^{+}$is a local ISS Liapunov-Krasovskii functional for system (11) if there exist two positive reals $k_{1}$ and $k_{2}, \mathcal{K}_{\infty}$-functions $a$ and $b$, and $\mathcal{K}$-functions $\chi$ and $\alpha$, such that, $\forall \varphi \in \mathcal{C}_{k_{1}}, \forall u$ with $|u|<k_{2}$ :

1) $a(|\varphi(0)|) \leq V(\varphi) \leq b\left(\|\varphi\|_{a}\right)$;

2) $D^{+} V(\varphi, u) \leq-\alpha\left(\|\varphi\|_{a}\right), \quad \forall\|\varphi\|_{a} \geq \chi(|u|)$.

Theorem 2.8: If system (11) admits a local ISS LiapunovKrasovskii functional, then it is locally ISS with $\gamma=a^{-1} \circ b \circ \chi$.

Definition 2.9: A locally Lipschitz continuous functional $V: \mathcal{C} \rightarrow$ $\mathrm{R}^{+}$is an ISS Liapunov-Krasovskii functional for system (11) if there exist $\mathcal{K}_{\infty}$-functions $a$ and $b$ and $\mathcal{K}$-functions $\chi$ and $\alpha$ such that:

1) $a(|\varphi(0)|) \leq V(\varphi) \leq b\left(\|\varphi\|_{a}\right)$

2) $D^{+} V(\varphi, u) \leq-\alpha\left(\|\varphi\|_{a}\right), \quad \forall\|\varphi\|_{a} \geq \chi(|u|)$.

Theorem 2.10: If system (11) admits an ISS Liapunov-Krasovskii functional, then it is ISS with $\gamma=a^{-1} \circ b \circ \chi$. 


\section{MAIN RESUlts}

\section{A. Links Between ISS and Exponential Stability}

Our main results can now be stated as follows.

Theorem 3.1: Let there exist positive reals $H, D$, and $\ell$ and a nonnegative real $p<1$, such that:

1) the unforced system (12) is exponentially stable (initial conditions in $\mathcal{C}_{H}$ );

2) for all $\varphi \in \mathcal{C}_{H}$, for all $u \in R^{m}$ with $|u|<D$, the following inequality holds:

$$
|f(\varphi, u)-f(\varphi, 0)| \leq \ell \max \left\{\|\varphi\|_{c}^{p}, 1\right\}|u| .
$$

Then, the perturbed system (11) is locally input-to-state stable, with $r$ and $r_{u}$ in Definition 2.5 being any positive reals satisfying the inequalities

$$
\begin{aligned}
& r<\frac{H}{A} \quad r_{u}<D \\
& \max \left\{\frac{2 C_{4} \ell r_{u}}{C_{3}},\left(\frac{2 C_{4} \ell r_{u}}{C_{3}}\right)^{(1 / 1-p)}\right\} \frac{C_{2}}{C_{1}}+\frac{C_{2}}{C_{1}} r<\frac{H}{A}
\end{aligned}
$$

$C_{i}, i=1,2,3,4$, being the positive constants given in Theorem 2.3, and $A$ being the constant in (3) for the unforced system (12).

Remark 2: A large class of systems verifies the condition (18). For instance, systems $\dot{x}(t)=f\left(x_{t}\right)+g\left(x_{t}\right) u(t)$, with any $f$ and $|g(\varphi)| \leq$ $\ell \max \left\{\|\varphi\|_{c}^{p}, 1\right\}$, verify the condition (18). The term $\max \left\{\|\varphi\|_{c}^{p}, 1\right\}$ may be substituted by a positive constant (included in $\ell$ ), but this would reduce, in general, the region where the ISS holds [see inequality (20)].

Theorem 3.2: Let system (12) be globally exponentially stable. Let there exist positive reals $L, \ell$ and a nonnegative real $p<1$ such that:

1) $\forall \varphi_{1}, \varphi_{2} \in \mathcal{C}$, the following inequality holds:

$$
\left|f\left(\varphi_{1}, 0\right)-f\left(\varphi_{2}, 0\right)\right| \leq L\left\|\varphi_{1}-\varphi_{2}\right\|_{c}
$$

2) $\forall \varphi \in \mathcal{C}, \forall u \in \mathrm{R}^{m}$, the following inequality holds:

$$
|f(\varphi, u)-f(\varphi, 0)| \leq \ell \max \left\{\|\varphi\|_{c}^{p}, 1\right\}|u| .
$$

Then, the perturbed system (11) is input-to-state stable.

\section{B. An Input-to-State Stabilizing Feedback}

In this section, we consider nonlinear systems

$$
\left\{\begin{array}{l}
\dot{x}(t)=f\left(x_{t}\right)+g\left(x_{t}\right)(u(t)+d(t)), \quad t \geq 0 \\
x_{0}=\psi,
\end{array}\right.
$$

where $f$ and $g$ are locally Lipschitz continuous functionals defined on $\mathcal{C}, f(0)=0, u(t) \in R^{m}$ is the control input, and $d(t) \in R^{m}$ is a measurable and locally essentially bounded disturbance. In recent literature (see [15]-[17], and [18]), many results concerning the input-output linearization and the elementary theory of nonlinear feedback for timedelay systems have been achieved. The following theorem provides an ISS feedback control law (see [2]) for the class of time-delay systems, which, by a suitable change of coordinates, state feedback control law, and in the case of no disturbance, can be transformed into a delay-free linear asymptotically stable system. Standard change of coordinates and feedback control laws, by which the closed-loop system, with no disturbance, becomes linear and delay-free, are considered. Such change of coordinates and feedback control laws exist for a significant class of time-delay systems.

Theorem 3.3: Consider system (23). Let there exist a diffeomorphism $\Phi: R^{n} \rightarrow R^{n}$ (the inverse is denoted with $\Phi^{-1}$ ), a locally Lipschitz functional $k: \mathcal{C} \rightarrow \mathcal{C}$, and a Hurwitz matrix $F \in R^{n \times n}$ such that: i) there exist suitable functions $L_{1}, L_{2}$, of class $\mathcal{K}_{\infty}$, such that the inequalities holds

$$
|\Phi(z)| \leq L_{1}(|z|) \quad\left|\Phi^{-1}(z)\right| \leq L_{2}(|z|) \quad \forall z \in R^{n} ;
$$

ii) with the change of coordinates $z=\Phi(x)$, system (23) is transformed into

$$
\begin{cases}\dot{z}(t)=\bar{f}\left(z_{t}\right)+\bar{g}\left(z_{t}\right)(u(t)+d(t)), & t \geq 0 \\ z_{0}(\theta)=\Phi(\psi(\theta)), & \theta \in[-\tau, 0] ;\end{cases}
$$

with $\bar{f}: \mathcal{C} \rightarrow R^{n}$ and $\bar{g}: \mathcal{C} \rightarrow R^{n \times m}$ locally Lipschitz functionals, such that, $\forall \varphi \in \mathcal{C}$, the following equality holds:

$$
\bar{f}(\varphi)+\bar{g}(\varphi) k(\varphi)=F \varphi(0) .
$$

Let $\Psi: \mathcal{C} \rightarrow \mathcal{C}$ be defined as

$$
\Psi(\varphi)(\theta)=\Phi(\varphi(\theta)), \quad \theta \in[-\tau, 0], \quad \varphi \in \mathcal{C} .
$$

Consider the feedback control law

$$
u(t)=k\left(\Psi\left(x_{t}\right)\right)-\bar{g}^{T}\left(\Psi\left(x_{t}\right)\right) Q \Phi(x(t))\left(\Phi(x(t))^{T} P \Phi(x(t))\right.
$$

where $P \in R^{n \times n}$ is any symmetric, positive-definite matrix and $Q \in$ $R^{n \times n}$ is the symmetric, positive-definite matrix solution of $F^{T} Q+$ $Q F=-P$.

Then, the closed-loop system (23)-(28) is input-to-state stable with respect to the measurable, locally essentially bounded disturbance $d(t)$.

Remark 3: Note that in this case the functionals $g$ and $\bar{g}$ do not have to satisfy the condition (18) as reported in Remark 2 . As is well known, given the symmetric, positive-definite matrix $P$, the matrix $Q$ is equal to $\int_{0}^{+\infty} e^{F^{T} t} P e^{F t} d t$.

Remark 4: If the change of coordinates and the feedback control law satisfying the hypotheses of Theorem 3.3 both exist in a neighborhood of the origin and in $\mathcal{C}_{H}$ for some positive real $H$, respectively, then, by the feedback control law (28), local ISS with respect to the disturbance is achieved. A local change of coordinates $\Phi$ and a local feedback control $k$ exist, for instance, for the class of nonlinear time-delay systems described by the equations (see [19])

$$
\dot{x}(t)=f(x(t))+g(x(t))\left[p_{1}\left(x_{t}\right)+p_{2}\left(x_{t}\right)(u(t)+d(t))\right]
$$

where the functions $f$ and $g$ are smooth, $f(0)=0$, and such as to admit full vector relative degree at 0 with respect to some smooth output function $h: R^{n} \rightarrow R^{m}, h(0)=0 ; p_{1}: \mathcal{C} \rightarrow R^{m}$ and $p_{2}: \mathcal{C} \rightarrow R^{m \times m}$ are locally Lipschitz functionals with $p_{1}(0)=0$, $\operatorname{det}\left(p_{2}(\phi)\right) \neq 0 \forall \phi \in \mathcal{C}_{H}, H$ positive real. Note that setting $u(t)=$ $\left(p_{2}\left(x_{t}\right)\right)^{-1}\left(-p_{1}\left(x_{t}\right)+v(t)\right)$ and considering $v(t)$ as a new input does not yield system (29) to be a finite-dimensional one, because of the standing term $p_{2}\left(x_{t}\right) d(t)$, which involves time delays. For instance, the classical Lotka-Volterra prey-predator system is described by equations (choosing the unknown variables as deviations from equilibrium) in the form (29) (see [20] and references therein). In this technical note, we have not considered maps from $\mathcal{C}$ to $R^{n}$ for the unknown variables in system (23), that is, $z(t)=\Phi\left(x_{t}\right)$ (see [17] and [18]), which may yield linearizing feedback control laws depending on both variables $z(t)$ and $x_{t}$, as well as internal dynamics described by coupled delay differential and difference equations. With such linearizing feedback control laws, further investigations concerning the ISS are necessary and will be a topic of future work.

\section{Proof of Theorem 3.1}

We view system (11) as a perturbation of the unforced system (12). The main idea of the proof will be to show that there exists a local ISS Liapunov-Krasovskii functional for system (11); Theorem 2.8 will 
then insure that our system is locally ISS. Moreover, the functional we are looking for is the same as the one for the unforced system (using Theorem 2.3). The details of the proof are reported below.

Proof: The converse Liapunov-Krasovskii Theorem 2.3 shows that the unforced system (12) has a Liapunov-Krasovskii functional $V(\varphi)$ that satisfies, in $\mathcal{C}_{(H / A)}$, the inequalities (5)-(7). Note that $V$ is Lipschitz in $\mathcal{C}_{(H / A)}$. Let $\|\varphi\|_{c}<(H / A)$ and $|u|<D$. Computing the upper right-hand side Dini derivative of the functional $V$ as in (15), we get

$$
\begin{aligned}
D^{+} & V(\varphi, u) \\
& =\limsup _{h \rightarrow 0} \frac{1}{h}\left(V\left(\varphi_{h, u}\right)-V(\varphi)\right) \\
& =\limsup _{h \rightarrow 0} \frac{1}{h}\left(V\left(\varphi_{h, u}\right)-V\left(\varphi_{h, 0}\right)-V(\varphi)+V\left(\varphi_{h, 0}\right)\right) \\
& \leq D^{+} V(\varphi, 0)+\underset{h \rightarrow 0}{\limsup } \frac{1}{h}\left(V\left(\varphi_{h, u}\right)-V\left(\varphi_{h, 0}\right)\right) \\
& \leq-C_{3}\|\varphi\|_{c}+\underset{h \rightarrow 0}{\limsup } \frac{1}{h}\left(V\left(\varphi_{h, u}\right)-V\left(\varphi_{h, 0}\right)\right) .
\end{aligned}
$$

Taking into account condition (18) on $f$, the following inequalities hold for sufficiently small $h$ :

$$
\begin{aligned}
& \left|V\left(\varphi_{h, u}\right)-V\left(\varphi_{h, 0}\right)\right| \\
& \quad \leq C_{4}\left\|\varphi_{h, u}-\varphi_{h, 0}\right\|_{c} \\
& \quad=C_{4} \sup _{s \in[-\tau, 0]}\left|\varphi_{h, u}(s)-\varphi_{h, 0}(s)\right| \\
& \quad=C_{4} \sup _{s \in[-h, 0]}|s+h||f(\varphi, u)-f(\varphi, 0)| \\
& \quad \leq C_{4}|h| \ell \max \left\{\|\varphi\|_{c}^{p}, 1\right\}|u| .
\end{aligned}
$$

Let $\omega: R^{+} \rightarrow R^{+}$be the class $\mathcal{K}_{\infty}$ function defined as $\omega(s)=\theta \min \left\{s, s^{1-p}\right\}$, where $0<\theta<\left(C_{3} / C_{4} \ell\right)$. Then, if $\|\varphi\|_{c} \geq \omega^{-1}(|u|)$, the following inequalities hold:

$$
D^{+} V(\varphi, u) \leq-C_{3}\|\varphi\|_{c}+C_{4} \ell \theta\|\varphi\|_{c} \leq-\delta\|\varphi\|_{c}
$$

where $\delta=C_{3}-C_{4} \ell \theta>0$. Let us choose $\theta=\left(C_{3} / 2 C_{4} \ell\right)$, so that $\delta=C_{3} / 2$. Hence, the conditions of Theorem 2.8 are satisfied using the norm $\|\cdot\|_{c}$ as a $\|\cdot\|_{a}$ seminorm, $a(s)=C_{1} s, b(s)=C_{2} s$, $\alpha(s)=\delta s$, and $\chi(s)=\omega^{-1}(s)$. We can conclude that system (11) is locally input-to-state stable and the inequality (13) holds, provided that the initial conditions satisfy $\|\varphi\|_{c}<r$ and the input $u(t)$ satisfies $\operatorname{ess~sup}_{t \geq 0}|u(t)|<r_{u}$, where $r$ and $r_{u}$ are suitable positive reals. From Theorem 2.8, it follows that the function $\gamma$ is given by

$$
\gamma(s)=\frac{C_{2}}{C_{1}} \max \left\{\frac{2 C_{4} \ell s}{C_{3}},\left(\frac{2 C_{4} \ell s}{C_{3}}\right)^{(1 / 1-p)}\right\} .
$$

Furthermore, from (30) and (5), it follows that the function $\beta$ in inequality (13) is given by

$$
\beta(s, t)=s \frac{C_{2}}{C_{1}} \exp \left(-\frac{C_{3}}{2} t\right) .
$$

The positive reals $r<(H / A)$ and $r_{u}<D$ can be computed by imposing

$$
\beta(r, 0)+\gamma\left(r_{u}\right)<\frac{H}{A}
$$

which, from (31) and (32), returns to (20).

The proof of Theorem 3.2 is similar and will not be detailed here.

\section{Proof of Theorem 3.3}

Proof: In the $z$ coordinates, the feedback control law (28) is given by

$$
u(t)=k\left(z_{t}\right)-\bar{g}^{T}\left(z_{t}\right) Q z(t) z(t)^{T} P z(t) .
$$

Let us apply Theorem 2.10 to the closed-loop system (in the new coordinates) (25)-(34), taking into account (26). Let us consider the Liapunov-Krasovskii functional $V(\varphi)=\varphi^{T}(0) Q \varphi(0)$. The following inequalities hold for $|d| \leq \varphi(0)^{T} P \varphi(0)$ :

$$
\begin{aligned}
D^{+} & V(\varphi, d) \\
= & \varphi^{T}(0)\left(Q F+F^{T} Q\right) \varphi(0) \\
& -2 \varphi^{T}(0) Q \bar{g}(\varphi) \bar{g}^{T}(\varphi) Q \varphi(0) \varphi^{T}(0) P \varphi(0)+2 \varphi^{T}(0) Q \bar{g}(\varphi) d \\
\leq & -\varphi^{T}(0) P \varphi(0)-2\left|g^{T}(\varphi) Q \varphi(0)\right|^{2} \varphi^{T}(0) P \varphi(0) \\
& +2\left|\varphi^{T}(0) Q \bar{g}(\varphi)\right||d|^{1 / 2}|d|^{1 / 2} \\
\leq & -\varphi^{T}(0) P \varphi(0)-2\left|g^{T}(\varphi) Q \varphi(0)\right|^{2} \varphi^{T}(0) P \varphi(0) \\
& +2\left|g^{T}(\varphi) Q \varphi(0)\right|^{2} \varphi^{T}(0) P \varphi(0)+\frac{1}{2} \varphi^{T}(0) P \varphi(0) \\
\leq & -\frac{1}{2} \varphi^{T}(0) P \varphi(0) .
\end{aligned}
$$

Therefore, by Theorem 2.10, it follows that the closed-loop system (in the new coordinates) (25)-(34) is input-to-state stable with respect to measurable and locally essentially bounded disturbances $d(t)$. Note that in this case the Euclidean norm $|\varphi(0)|$ is used as a $\|\varphi\|_{a}$ seminorm.

So, there exist a function $\beta_{z}$ of class $\mathcal{K} \mathcal{L}$ and a function $\gamma_{z}$ of class $\mathcal{K}$ such that the following inequality holds $\forall t \geq 0$ :

$$
|z(t)| \leq \beta_{z}\left(\left\|z_{0}\right\|, t\right)+\gamma_{z}\left(\left\|d_{[0, t)}\right\|_{\infty}\right) .
$$

From (35), taking into account (24), it follows that

$$
\begin{aligned}
|x(t)| & =\left|\Phi^{-1}(z(t))\right| \\
& \leq L_{2}\left(\beta_{z}\left(\left\|\Psi\left(x_{0}\right)\right\|_{\infty}, t\right)+\gamma_{z}\left(\left\|d_{[0, t)}\right\|_{\infty}\right)\right) \\
& \leq L_{2}\left(2 \beta_{z}\left(L_{1}\left(\left\|x_{0}\right\|_{\infty}\right), t\right)\right)+L_{2}\left(2 \gamma_{z}\left(\left\|d_{[0, t)}\right\|_{\infty}\right)\right) .
\end{aligned}
$$

Because the functions $(s, t) \rightarrow L_{2}\left(2 \beta_{z}\left(L_{1}(s), t\right)\right), s \rightarrow L_{2}\left(2 \gamma_{z}(s)\right)$, $(s, t) \in R^{+} \times R^{+}$, are of class $\mathcal{K} \mathcal{L}$ and of class $\mathcal{K}$ respectively, the ISS of the closed-loop system (23)-(28) is proved.

\section{EXAMPLES}

Theorems 3.1, 3.2, and 3.3 have nice applications in the nonlinear feedback control of time-delay systems when a disturbance adds to the control law, which usually happens because of actuator errors.

As an application of Theorem 3.2, consider the following time-delay system:

$$
\left\{\begin{array}{l}
\dot{x}_{1}(t)=x_{2}(t)+\int_{-1}^{0} 0.1 \theta x_{1}(t+\theta) d \theta \\
\dot{x}_{2}(t)=x_{1}(t-1) x_{2}(t-1) \\
\quad+\left(1+\left|x_{1}(t-1)\right|^{(1 / 2)}\right)(u(t)+d(t))
\end{array}\right.
$$

where $u$ is the control input and $d$ is an unknown measurable, locally essentially bounded disturbance. The following control law:

$$
u(t)=\frac{-x_{1}(t-1) x_{2}(t-1)-2 x_{1}-3 x_{2}}{1+\left|x_{1}(t-1)\right|^{1 / 2}}
$$

is such that the closed-loop system becomes

$$
\left\{\begin{array}{l}
\dot{x}_{1}(t)=x_{2}(t)+\int_{-1}^{0} 0.1 \theta x_{1}(t+\theta) d \theta \\
\dot{x}_{2}(t)=-2 x_{1}(t)-3 x_{2}(t)+\left(1+\left|x_{1}(t-1)\right|^{(1 / 2)}\right) d(t) .
\end{array}\right.
$$

The closed-loop system (37) with zero disturbance $(d(t) \equiv 0)$ is a globally exponentially stable linear time-delay system (it can be 
checked by [21, Proposition 5.15, pp. 171]). For this example, the condition (22) is respected. The following inequality holds, for any $\varphi=\left[\begin{array}{ll}\varphi_{1} & \varphi_{2}\end{array}\right]^{T} \in \mathcal{C}$ (with $n=2$ ):

$$
\begin{aligned}
|f(\varphi, d)-f(\varphi, 0)| & =\left(1+\left|\varphi_{1}(-1)\right|^{(1 / 2)}\right)|d| \\
|d|+\left|\varphi_{1}(-1)\right|^{(1 / 2)}|d| & \leq 2 \max \left\{\|\varphi\|_{c}^{(1 / 2)}, 1\right\}|d| .
\end{aligned}
$$

Therefore, Theorem 3.2 allows us to say that the closed-loop system (37) has the important ISS property with respect to the disturbance $d(t)$.

As an application of Theorem 3.3, consider the following time-delay system:

$$
\left\{\begin{array}{l}
\dot{x}_{1}(t)=x_{1}^{2}(t)+x_{2}(t) \\
\dot{x}_{2}(t)=x_{1}(t-\tau) x_{2}(t-\tau) \\
\quad+\left(1+x_{1}^{2}(t) x_{2}^{2}(t-\tau)\right)(u(t)+d(t))
\end{array}\right.
$$

where $u$ is the control input and $d$ is an unknown measurable, locally essentially bounded disturbance. Consider the change of coordinates [17], [18] $z=\left[\begin{array}{ll}z_{1} & z_{2}\end{array}\right]^{T}=\Phi(x)=\left[\begin{array}{ll}x_{1} & x_{1}^{2}+x_{2}\end{array}\right]^{T}$. Then, $\Psi$ is defined as, for $\varphi=\left[\begin{array}{ll}\varphi_{1} & \varphi_{2}\end{array}\right]^{T} \in \mathcal{C}, \Psi(\varphi)(\theta)=\left[\begin{array}{c}\varphi_{1}(\theta) \\ \varphi_{1}^{2}(\theta)+\varphi_{2}(\theta)\end{array}\right]$, $\theta \in[-\tau, 0]$. By this change of coordinates, system (4) is transformed into system (25) with $\bar{f}$ and $\bar{g}$ given by

$$
\begin{aligned}
& \bar{f}(\varphi)=\left[\begin{array}{c}
\varphi_{2}(0) \\
2 \varphi_{1}(0) \varphi_{2}(0)+\varphi_{1}(-\tau)\left(\varphi_{2}(-\tau)-\varphi_{1}^{2}(-\tau)\right)
\end{array}\right] \\
& \bar{g}(\varphi)=\left[\begin{array}{c}
0 \\
1+\varphi_{1}^{2}(0)\left(\varphi_{2}(-\tau)-\varphi_{1}^{2}(-\tau)\right)^{2}
\end{array}\right] .
\end{aligned}
$$

Let $k: \mathcal{C} \rightarrow \mathcal{C}$ be defined as [17], [18]

$$
\begin{aligned}
k(\varphi)=\frac{-2 \varphi_{1}(0) \varphi_{2}(0)-\varphi_{1}(-\tau)\left(\varphi_{2}(-\tau)-\varphi_{1}^{2}(\tau)\right)}{1+\varphi_{1}^{2}(0)\left(\varphi_{2}(-\tau)-\varphi_{1}^{2}(-\tau)\right)^{2}} & \\
& +\frac{r_{1} \varphi_{1}+r_{2} \varphi_{2}}{1+\varphi_{1}^{2}(0)\left(\varphi_{2}(-\tau)-\varphi_{1}^{2}(-\tau)\right)^{2}}
\end{aligned}
$$

with $r_{1}$ and $r_{2} \in R$. By this choice of $k$, it follows that $\bar{f}(\varphi)+$ $\bar{g}(\varphi) k(\varphi)=F \varphi(0)$, where $F=\left[\begin{array}{cc}0 & 1 \\ r_{1} & r_{2}\end{array}\right]$. By an easy choice of $r_{1}$ and $r_{2}$, the matrix $F$ is Hurwitz.

By Theorem 3.3, the following control law:

$$
u(t)=k\left(\Psi\left(x_{t}\right)\right)-\bar{g}\left(\Psi\left(x_{t}\right)\right) Q \Phi(x(t)) \Phi(x(t))^{T} \Phi(x(t))
$$

where $\Phi$ and $\Psi$ are defined above, $Q$ is the symmetric positive matrix satisfying

$$
\left[\begin{array}{ll}
0 & r_{1} \\
1 & r_{2}
\end{array}\right] Q+Q\left[\begin{array}{cc}
0 & 1 \\
r_{1} & r_{2}
\end{array}\right]=-I
$$

and $I$ is the identity matrix in $R^{2 \times 2}$, is such that the closed-loop system (40) and (41) is (globally) ISS with respect to the disturbance $d(t)$. Note that the functionals $g$ and $\bar{g}$ are (only) locally Lipschitz and that system (40) rewritten in the new coordinates, with $u(t)=k\left(z_{t}\right)$ and zero disturbance $(d(t) \equiv 0)$ is a globally exponentially stable (linear) delay-free system. However, if the disturbance is present, the time-delay closed-loop system in the new coordinates is not ISS. Actually, any constant disturbance may cause the variables $z(t)$ (and, consequently, the variables $x(t)$ ) to go to $\infty$ for suitable initial conditions. Note also that the condition (22) is not verified for system (40), rewritten in the new coordinates, in closed loop with $u(t)=k\left(z_{t}\right)$. For this, just take into account remark (2) and that

$$
|\bar{g}(\varphi) d|=\left|1+\varphi_{1}^{2}(0)\left(\varphi_{2}(-\tau)-\varphi_{1}^{2}(-\tau)\right)^{2}\right||d| .
$$

By choosing, for instance, all $\varphi=\left[\begin{array}{c}\varphi_{1} \\ 0\end{array}\right]$ with $\varphi_{1}$ a constant function, one obtains $|\bar{g}(\varphi) d| \geq\|\varphi\|_{c}^{6}|d|$. Therefore, a positive real $l$ and a nonnegative real $p<1$, such that $|\bar{g}(\varphi) d| \leq l\|\varphi\|_{c}^{p}|d|$ holds for all above $\varphi$ with the constant value greater than 1 , do not exist.

\section{CONCLUSION}

The ISS theory, recently being adapted to time-delay systems, is one of the best tools for analysis and control of nonlinear systems. In this technical note, we establish a connection between ISS and exponential stability of time-delay systems when the chosen functional verifies a Lipschitz-like hypothesis with respect to the input. Roughly speaking, it is proved that a system which is exponentially stable in the unforced case is input-to-state stable when it is forced by a bounded input. A second task has also been fulfilled: a new feedback control law is provided for delay-free linearizable and stabilizable time-delay systems. With the given feedback law, the closed-loop system is ISS with respect to disturbances adding to the control law.

These results are important for several main reasons. On the one hand, one has a new opportunity to check the ISS property of delayed systems. The given Lipschitz-like hypothesis allows us to consider a large class of time-delay systems and it has to be said that, even in the nondelayed case, the only existing result linking ISS and exponential stability is for systems which are globally Lipschitz (see, for instance, [22]). Nevertheless, it is true that at the moment, we do not have a tool to check if the following Lipschitz-like condition is the most accurate one. Further work can be done in this direction. On the other hand, the feedback control law allows us to deal with a typical problem in applied systems: having this new feedback, one can avoid the actuator errors as the closed-loop system is ISS with respect to disturbances adding to the control law. This is of course a first step in the direction because the feedback linearization is still a hard task for nonlinear delayed systems.

\section{REFERENCES}

[1] E. Sontag, "The ISS philosophy as a unifying framework for stabilitylike behavior," in Proc. Nonlinear Control in the Year 2000, 2000, vol. 2, pp. 443-468.

[2] E. Sontag, "Smooth stabilization implies coprime factorization," IEEE Trans. Autom. Control, vol. 34, no. 4, pp. 435-443, Apr. 1989.

[3] A. Teel, "Connections between Razumikhin-type theorems and the ISS nonlinear small gain theorem," IEEE Trans. Autom. Control, vol. 43, no. 7, pp. 960-964, Jul. 1998.

[4] P. Pepe and Z.-P. Jiang, "A Lyapunov-Krasovskii methodology for ISS and iISS of time-delay systems," Syst. Control Lett., vol. 55, no. 12, pp. 1006-1014, 2006.

[5] D. Liberzon, "Quantization, time delays, and nonlinear stabilization," IEEE Trans. Autom. Control, vol. 51, no. 7, pp. 1190-1195, Jul. 2006.

[6] I. Polushin and H. Marquez, "Stabilization of bilaterally controlled teleoperators with communication delay: An ISS approach," Syst. Control Lett., vol. 24, pp. 351-359, 2003.

[7] N. Yeganefar, M. Dambrine, and N. Yeganefar, "Relation between exponential stability and input-to-state stability for time-delay systems," in Proc. Autom. Control Conf., New York, NY, 2007, pp. 4919-4920.

[8] E. Mazenc, M. Michael, and L. Zongli, "On input-to-state stability for nonlinear systems with delayed feedbacks," in Proc. Autom. Control Conf., New York, NY, 2007, pp. 4804-4809.

[9] N. Krasovskii, Stability of Motion. Stanford, CA: Stanford Univ. Press, 1963.

[10] R. Driver, "Existence and stability of solutions of a delay-differential system," Arch. Rational Mech. Anal., vol. 10, pp. 401-426, 1962.

[11] T. Burton, Stability and Periodic Solutions of Ordinary and Functional Differential Equations. New York: Academic, 1985.

[12] I. Karafyllis, "Lyapunov theorems for systems described by retarded functional differential equations," Nonlinear Anal.: Theory Methods Appl., vol. 64, no. 3, pp. 590-617, 2006.

[13] P. Pepe, "On Lyapunov-Krasovskii functionals under carathéodory conditions," Automatica, vol. 43, no. 4, pp. 701-706, 2007.

[14] P. Pepe, "The problem of the absolute continuity for LiapunovKrasovskii functionals," IEEE Trans. Autom. Control, vol. 52, no. 5, pp. 953-957, May 2007.

[15] L. Marquez-Martinez and C. Moog, "Input-output feedback linearization of time-delay systems," IEEE Trans. Autom. Control, vol. 49, no. 5, pp. 781-786, May 2004.

[16] L. Marquez-Martinez and C. Moog, "New insights on the analysis of nonlinear time-delay systems: Application to the triangular equivalence," Syst. Control Lett., vol. 56, pp. 133-140, 2007.

[17] T. Oguchi, A. Watanabe, and T. Nakamizo, "Input-output linearization of retarded non-linear systems by using an extension of lie derivative," Int. J. Control, vol. 75, no. 8, pp. 582-590, 2002. 
[18] A. Germani, C. Manes, and P. Pepe, "Input-output linearization with delay cancellation for nonlinear delay systems: The problem of the internal stability," Int. J. Robust Nonlinear Control, vol. 13, no. 9, pp. 909-937, 2003, Special Issue on Time Delay Systems.

[19] P. Pepe, "Preservation of the full relative degree for a class of delay systems under sampling," ASME J. Dyn. Syst. Meas. Control, vol. 125, no. 2, pp. 267-270, 2003.

[20] P. Pepe, "Adaptive output tracking for a class of nonlinear time delay systems," Int. J. Adapt. Control Signal Process., vol. 18, no. 6, pp. 489-503, 2004.

[21] K. Gu, V. Kharitonov, and J. Chen, Stability of Time-Delay Systems. Boston, MA: Birkhauser, 2003.

[22] H. Khalil, Nonlinear Systems, 3rd ed. Englewood Cliffs, NJ: Prentice-Hall, 2002.

\section{Off-Line Reference Shaping of Periodic Trajectories for Constrained Systems With Uncertainties}

Hiromi Suzuki, Member, IEEE, and Toshiharu Sugie, Fellow, IEEE

\begin{abstract}
This paper is concerned with off-line reference shaping of infinite horizon periodic trajectories for uncertain closed-loop systems with state/input constraints. The method allows us to improve tracking performance subject to constraints in the presence of uncertainties. First, the reference shaping for nominal systems is proposed, which yields a suboptimal solution through decomposition of the infinite horizon tracking problem into two finite ones. Second, the method is extended to fulfill the constraints robustly.
\end{abstract}

Index Terms-Feed-forward predictive control, infinite horizon control, model uncertainty, state and control constraints, trajectory tracking.

\section{INTRODUCTION}

Most real plants have some constraints on their state and/or input such as actuator saturation and amplitude limitation of certain states. Without taking these constraints into account, we may have wind-up phenomena and/or serious performance degradation. One approach to overcome this issue is to modify the reference signals in such a way that the system constraints are satisfied, which is known as a reference governor approach [1]-[3]. The approach inherently prevents the system from violating constraints because one of the major factors of constraint violation is to inject inappropriate reference signals. In addition, it is rather simple and applicable to existing control systems.

Unfortunately, since most of the existing works discuss on-line reference modification, it is difficult to improve tracking performance, and heavy computation burden may become a problem. In some practical cases, however, reference signals are given in advance; it is NOT necessary to modify the reference on-line in such cases. Based on this observation, a pure feed-forward approach, which generates new reference signals through off-line computation, has been proposed to achieve better tracking performance for constrained linear systems [4]. According to various numerical examples, this method improves the tracking performance drastically. It can be regarded as nonlinear

Manuscript received September 4, 2006; revised January 8, 2007, September 5, 2007, and February 6, 2008. Current version published August 29, 2008. Recommended by Associate Editor M. Kothare.

The authors are with the Department of Systems Science, Graduate School of Informatics, Kyoto University, Kyoto 611-0011, Japan (e-mail: suzuki@ robot. kuass.kyoto-u.ac.jp; sugie@ robot.kuass.kyoto-u.ac.jp).

Color versions of one or more of the figures in this paper are available online at http://ieeexplore.ieee.org.

Digital Object Identifier 10.1109/TAC.2008.928310

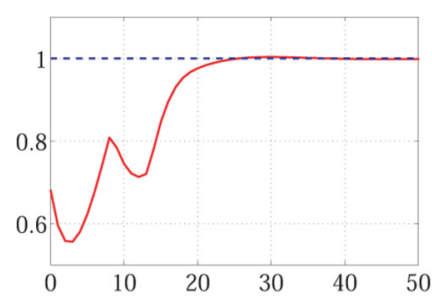

(a) Shaped reference: $r(k)$

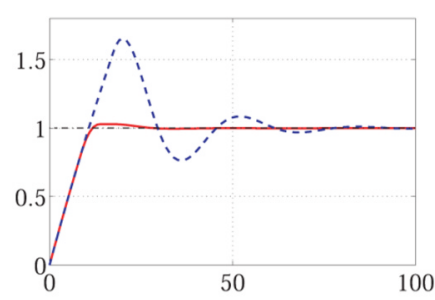

(b) Output: $y(k)$
Fig. 1. Step trajectory tracking with reference shaping.

two-degree-of-freedom control. This work was followed in [5], where the constraint fulfillment is guaranteed in infinite horizon cases.

The purpose of this note is to establish a method of shaping the periodic reference trajectories with reasonable computation burden so that the tracking performance will be improved without violating the constraints on state and/or input in the presence of model uncertainties. The method optimizes the nominal tracking performance subject to a robust constraint fulfillment condition.

NOTATION: Let $M_{1}, M_{2} \in \mathbf{R}^{m \times n}$ and $x, y \in \mathbf{R}^{n} . x^{i}$ denotes the $i$-th component of $x . M^{i, j}$ denotes the $(i, j)$-th component of $M$. Inequalities hold component-wise, i.e., $M_{1}>M_{2}$ implies that $M_{1}^{i, j}>$ $M_{2}^{i, j}, \forall i, j$.

\section{BASIC CONCEPT OF REFERENCE SHAPING}

This section provides an introductory view of reference shaping: The concept of the method and its effectiveness are briefly demonstrated through a motivating example. This is followed by the system description and problem formulation.

\section{A. How Does the Reference Shaping Work?}

Consider the internally stable unity feedback system which consists of $P(z)=(0.00662 / z-0.987)$ and $C(z)=(22 z-16 / z-1)$. Assume the system has the input saturation constraint $|u(k)| \leq 15$. Our motivation is to achieve better tracking performance without violating this constraint.

The simulation result in Fig. 1 illustrates the effectiveness of the reference shaping. The output $y(k)$ is supposed to track $r_{0}(k)=1$ quickly. Fig. 1(a) and (b) show the reference signal $r(k)$ and the plant output $y(k)$, respectively. First, we apply the step reference signal $r(k)=1$. The dashed line in Fig. 1(b) shows the corresponding output. A big overshoot occurs in terms of the input saturation.

However, the shaped reference signal, shown by the bold line in Fig. 1(a), avoids such performance degradation: The corresponding output is given by the bold line in Fig. 1(b), which shows that the tracking property is drastically improved. This reference signal is obtained by the off-line reference shaping method we are proposing in this paper. The above shaped signal looks strange, but it achieves satisfactory tracking performance without constraint violation.

Now, let us make a few remarks on the difference from the existing reference governor approaches.

- It is not possible for reference governors to improve tracking performance when the original reference signal does not cause constraint violation, while the above off-line shaping can.

- On-line computation burden is negligible in the proposed method, contrary to reference governors. Therefore, it can be easily applied to systems with fast sampling rates such as electromechanical systems.

\section{B. System Description and Problem Formulation}

Consider the linear discrete-time closed-loop system $\Sigma$ which consists of the plant and its stabilizing controller. The closed-loop system 